\title{
Apoyo al Ciudadano desde la Extensión Universitaria para la Accesibilidad e Inclusión Digital
}

\author{
Raúl J. Martelo(1) Iris A. Jiménez ${ }^{(2)}$ y José D.C. Jaimes ${ }^{(3) *}$ \\ (1) Univ. de Cartagena, Fac. de Ingeniería, Grupo de Investigación en Tecnologías de las \\ Comunicaciones e Informática (GIMATICA), Avenida el Consulado, Calle 30 No. 48-152. Cartagena, \\ Bolívar-Colombia. \\ (2) Universidad de la Guajira, Facultad de Ciencias Básicas y Aplicadas, Grupo de Investigación \\ BIEMARC, Km 5 vía a Maicao, Guajira-Colombia. \\ (3) Universidad de Cartagena, Facultad de Ingeniería, Grupo de Investigación en Medio Ambiente, \\ Alimentos y Salud (MAAS), Cra. 50 \#24-120, Cartagena, Bolívar- Colombia. (e-mail: \\ jjaimesmor@yahoo.es) \\ ${ }^{*}$ Autor a quien debe ser dirigida la correspondencia
}

Recibido Oct. 12, 2016; Aceptado Dic. 14, 2016; Versión final Ene. 10, 2017, Publicado Jun. 2017

\begin{abstract}
Resumen
En la presente investigación se realiza una revisión sobre el papel de las universidades colombianas, desde la extensión, para aumentar los niveles de accesibilidad e integración digital de los ciudadanos, con la finalidad de incrementar su relación con los factores clave de la sociedad: gobierno, educación y empresas. La investigación se tipificó como descriptiva con un diseño transeccional-descriptivo y de campo. La recolección de información se realizó a través de un cuestionario, el cual fue validado y su confiabilidad fue calculada con el coeficiente Alfa de Crombach, resultando en 0,85. El mismo estuvo conformado por nueve preguntas, con una escala de cinco opciones de respuestas. La muestra fue de tipo probabilística, conformada por 400 sujetos pertenecientes al Departamento de la Guajira. Finalmente, se concluyó que la mayoría de los sujetos consultados estuvo de acuerdo con que la universidad lidere la formación de los ciudadanos para mejorar sus niveles de accesibilidad e inclusión digital.
\end{abstract}

Palabras clave: accesibilidad; inclusión digital; extensión; educación; empresas; gobierno local

\section{Support to Citizens through University Extension Programs for Digital Inclusion and Accessibility}

\begin{abstract}
This research reviews the role of extension programs at Colombian universities aimed to increasing the levels of accessibility and digital integration of citizens, with the purpose of improving their relationship with key factors of society: government, education and companies. The research was classified as descriptive with a transectional-descriptive and field design. Data were collected by means of a survey, which was validated by experts. Its reliability was calculated with the Cronbach's alpha coefficient, resulting to be 0.85 . The questionnaire consisted of nine questions, with a Likert scale of five choices. The probabilistic-type sample was formed by 400 subjects from the Department of La Guajira. The main conclusion is that most of the subjects agree that universities should lead training processes addressed to citizens for increasing their levels of accessibility and digital inclusion.
\end{abstract}

Keywords: accessibility; digital inclusion; extension; education; business; local government 


\section{INTRODUCCIÓN}

Sequera (2011) afirma que la extensión universitaria está ligada a una gama de "propósitos, ámbitos, programas, prácticas y acciones que se ejecutan bajo su denominación" entre los que se pueden mencionar: actividades de difusión y divulgación cultural, actividades de servicio social voluntario u obligatorio (estudiantes), programas de educación continua, programas de vinculación con organizaciones sociales y otras entidades públicas y privadas, servicios universitarios(clínicas, consultoría, gestión), entre otros. En la extensión se agruparían todas aquellas actividades que no son propiamente docencia ni investigación. En este contexto, el propósito de esta investigación, es determinar cuál debería ser el papel de las universidades colombianas para aumentar los niveles de accesibilidad e integración digital de los ciudadanos, en función de incrementar su relación con los factores clave de la sociedad: gobierno, educación y empresas. La integración del ciudadano con estos factores claves, garantizaría el crecimiento del país de manera equitativa, así cada uno de los ciudadanos tendría igualdad de oportunidades, lo que refuerza la democracia en la sociedad. En este sentido, la extensión universitaria realiza una de las funciones sustantivas de la academia, como es la creación y transmisión de conocimientos.

Ortiz y Morales (2011) afirman que el conocimiento es un factor clave para la generación de ventajas competitivas. Tal afirmación refuerza el rol protagónico de las universidades, específicamente la función de la extensión universitaria puesto que es la que permite interactuar directamente con el entorno. Para profundizar en este contexto, es importante resaltar el papel de las tecnologías de información y comunicación (TIC) en este proceso, ya que su crecimiento ha dado pie a un cambio social, principalmente en la manera cómo se manejan actualmente las comunicaciones, lo que modifica los puntos de vista de los diferentes fenómenos sociales. La comunicación instantánea en tiempo real es uno de los avances más importantes del uso de las TIC. Actualmente, se puede tener acceso a la información de manera inmediata en el momento que sucede algún evento. Esto ha cambiado la forma cómo los medios de comunicación transmiten las noticias; se ha pasado de un rotativo impreso, que contiene las noticias de ayer, al uso de portales, donde la noticia es casi en tiempo real.

Igualmente, se utilizan las TIC en otros procesos que facilitan las gestiones que realizan los ciudadanos, como por ejemplo, las transacciones bancarias como transferencias o pagos de servicios, que se pueden realizar desde la casa u oficina, sin necesidad de moverse hasta la entidad bancaria, o hasta el lugar donde se pagan los servicios, representando un ahorro sustancial de tiempo y recursos que pueden ser utilizados en actividades adicionales. Los ciudadanos pueden, desde la comodidad de sus hogares, resolver casi cualquier problema o encontrar información importante. En el ámbito educativo, el uso de las TIC ha fortalecido algunos procesos. Actualmente, existen cursos de todo tipo, inclusive estudios universitarios, a los cuales se puede acceder de manera digital y a distancia y donde es posible obtener la capacitación que se desee. Al hacer una búsqueda en internet, se observa como universidades como la Internacional de la Rioja, Universidad a Distancia de Madrid - UDIMA y la Universidad Católica de Ávila - UCAV en España, la EAN en Colombia y muchas otras en Estados Unidos, imparten estudios en todos los niveles en el ámbito nacional e internacional.

Antes de continuar, es imperativo aclarar el significado de las variables accesibilidad e inclusión digital. Para el proyecto Living Lab de la Universidad de Antioquia (2014), la accesibilidad digital significa que las personas con discapacidad (visual, auditiva, física, del habla, cognitivas y alteraciones neurológicas) pueden percibir, entender, navegar e interactuar con la web y sus contenidos. Cuando ello se da, cumple con el objetivo para el cual fue concebida y el impacto de la discapacidad disminuye radicalmente, pues se eliminan las barreras para la comunicación e interacción que enfrentan muchas personas en el mundo físico.

Sin embargo, para García et al., (2008), el concepto de accesibilidad hacía referencia únicamente a las mejoras en los entornos físicos, específicamente, a la eliminación de las barreras arquitectónicas. Actualmente, se entiende por accesibilidad al conjunto de medidas muy diversas que se deben considerar en la elaboración de las estrategias políticas o sociales, de modo que la accesibilidad sea universal o integral. Para estos autores, un entorno es plenamente accesible cuando se puede avanzar en todos sus caminos de forma autónoma. En este sentido, los mismos autores se refieren a la accesibilidad web o accesibilidad digital como la capacidad que tienen todas las personas, sin importar la discapacidad que presenten, sea física, intelectual o técnica, de acceder a los contenidos de la internet. Estos autores manejan un amplio concepto del término discapacidad, relacionándolo no solo con el aspecto funcional y orgánico de los individuos, sino que amplían su significado con lo relativo a los aspectos técnicos, por lo que al referirse a una personas con discapacidad, no solo se refiere a aquellas que presentan problemas físicos o intelectuales, sino que engloban a personas sanas sin acceso a recursos técnicos, lo que a su vez, incluye el conocimiento que puedan tener de las TIC.

En cuanto a la inclusión digital, según Agustín y Clavero (2009), es un concepto acuñado en el año 2003 por los grupos ciudadanos que impulsaron la campaña para promover los Derechos de Comunicación en la 
Sociedad de la Información, más conocida por el acrónimo que corresponde a su denominación en lengua inglesa Communication Rights in the Information Society (CRIS), durante el desarrollo de la primera fase de la Cumbre Mundial de la Sociedad de la Información celebrada en Ginebra. Estos autores conciben la inclusión digital como una forma de inserción social cuyo concepto es el contrapuesto al de brecha digital. Consideran que la inclusión digital está conformada por las políticas públicas relacionadas con la construcción, administración, expansión, ofrecimiento de contenidos y desarrollo de capacidades locales en las redes digitales públicas, alámbricas e inalámbricas, respetando las garantías de privacidad y seguridad de manera equitativa para todos los ciudadanos.

Para Ramírez et al., (2010), el concepto de inclusión digital, es la antítesis a la exclusión generada por el acceso desigual y fragmentado a las TIC, que a su vez, impide la participación y el ejercicio ciudadano. Expresan, de igual manera, que la inclusión digital es un proceso que permite a los ciudadanos integrarse a la sociedad. Cabezudo (2011) manifiesta que no se trata solamente de tener acceso a la tecnología, o de la conexión a internet, se trata además de contar con una capacitación para operar estos equipos. Por su parte Torres (2015) manifiesta que es un derecho complejo, que a pesar de estar en formación, debe tratarse como un derecho central, que contribuye con el fortalecimiento de las democracias, en el cual se reconoce no solamente el auge tecnológico, sino también la existencia de brechas y desigualdades. En este sentido, es un derecho social, el cual el estado debe garantizar su acceso a todos los ciudadanos.

Para López y Samek (2009), la Inclusión digital es un derecho humano que presenta una serie de obstáculos como la pobreza informativa, la censura, el uso político de las tecnologías, la desinformación, la manipulación de los medios de comunicación y la destrucción de información pública. La idea es presionar para la creación, desarrollo y experimentación de proyectos de inclusión digital crítica que incluyan a las personas a menudo excluidas de la sociedad, marginados o negados por factores como su sexo, identidad de género, clase, orientación sexual, nacionalidad, origen étnico, ubicación geográfica, idioma, patrimonio, capacidad física o mental, educación, creencias, filosofía política, circunstancias económicas, raza, y/o religión. Así, pues, la inclusión digital, puede fomentar nuevos espacios para la tolerancia y la comprensión y contrarrestar los intentos de personas y grupos que buscan imponer valores, costumbres o creencias en el mundo digital y, que, en última instancia, afectan también a zonas no conectadas.

Por su parte, Duarte (2011) relaciona las TIC con la inclusión, al expresar que estas alteran la intensidad y la forma de las relaciones interpersonales e institucionales; esto debido principalmente a que las TIC representan la oportunidad de incluir a millones de individuos en el acceso y comunicación con los servicios del Estado e instituciones privadas y en la inserción al campo laboral. Este autor propone tres categorías conceptuales para el análisis de los proyectos de inclusión digital: la conectividad, la accesibilidad y comunicabilidad. Según el mismo autor, la conectividad se refiere a la oferta y a la provisión de infraestructura y de equipos que permiten la conexión tecnológica a la red global de tecnologías de información y comunicación o internet. La accesibilidad se refiere a los programas que permiten la apropiación social de las infraestructuras y equipos tecnológicos, la cual se centra en la capacitación tecnológica. Por último, la comunicabilidad hace referencia al uso de las TIC sin impedimentos, de manera que su uso permita influir e incluso modificar, las relaciones socioeconómicas, políticas y culturales de los actores involucrados.

Por otra parte, en opinión de Mochi (2012), la inclusión digital supone poder desarrollar capacidades individuales y territoriales para la adquisición de técnicas y habilidades en personas que han tenido muy poco contacto con las TIC y expone que el principal reto del Estado no es sólo garantizar el acceso público a las TIC para los sectores más vulnerables de la población, sino ofrecer un acceso participativo, universal, democrático e inclusivo que, en el futuro, contribuirá con la reducción de las brechas sociales. En el portal Colombia Digital (2016) se presenta el proceso creciente de digitalización como el factor que está generando una profunda transformación en la manera de relacionarse, aprender, educar, trabajar, liderar personas, proyectos y organizaciones, y su influencia en los ámbitos personales, públicos y profesionales, aceptando la incidencia de las TIC en el aula y en los centros educativos.

En los párrafos anteriores se puede observar que la relación entre accesibilidad e inclusión digital, no existe una sin la otra; sin accesibilidad no hay inclusión digital y sin inclusión digital no puede existir la accesibilidad.

\section{LA SITUACION DE COLOMBIA}

En el Reporte Global de Tecnologías de la Información (DNP, 2015), en lo referente a los resultados obtenidos por Colombia, muestran que la calificación del Índice de Preparación para la Conectividad (IPC) obtenida por Colombia fue 4,1 en una escala de uno a siete, ubicándose en el puesto 64 entre 143 países a nivel mundial. Sin embargo, esta posición representa un descenso de una posición en relación con el resultado obtenido en el año 2014, cuando con una calificación de 4,05 el país había ocupado la posición 63 entre 148 países. Teniendo en cuenta la disminución de cinco en el número de países analizados frente al ejercicio del año 
2014, en la Figura 1 se puede observar que Colombia disminuyó su posición relativa, la cual pasó de 57,4 por ciento en 2014 a 55,2. La posición perdida por Colombia se explica por la recomposición del grupo de países que la preceden, la cual resulta de: (i) Armenia y Rumania se colocan por encima de Colombia en el escalafón al pasar de las casillas 65 a 58 y 75 a 63, respectivamente; (ii) Brunei, que el año anterior ocupaba la posición 45 no fue incluido este año.

En la Figura 1 (ITU, 2015), se muestra como ha sido la evolución de Colombia en el crecimiento del índice IPC desde el año 2001. Al analizar todo el informe en cuestión, se puede observar que pese a los esfuerzos tanto del Estado como del sector privado y educativo, todavía falta mucho por hacer con respecto a la reducción de la brecha digital en Colombia, lo que incide directamente en la mejora de los índices de accesibilidad e inclusión digital.

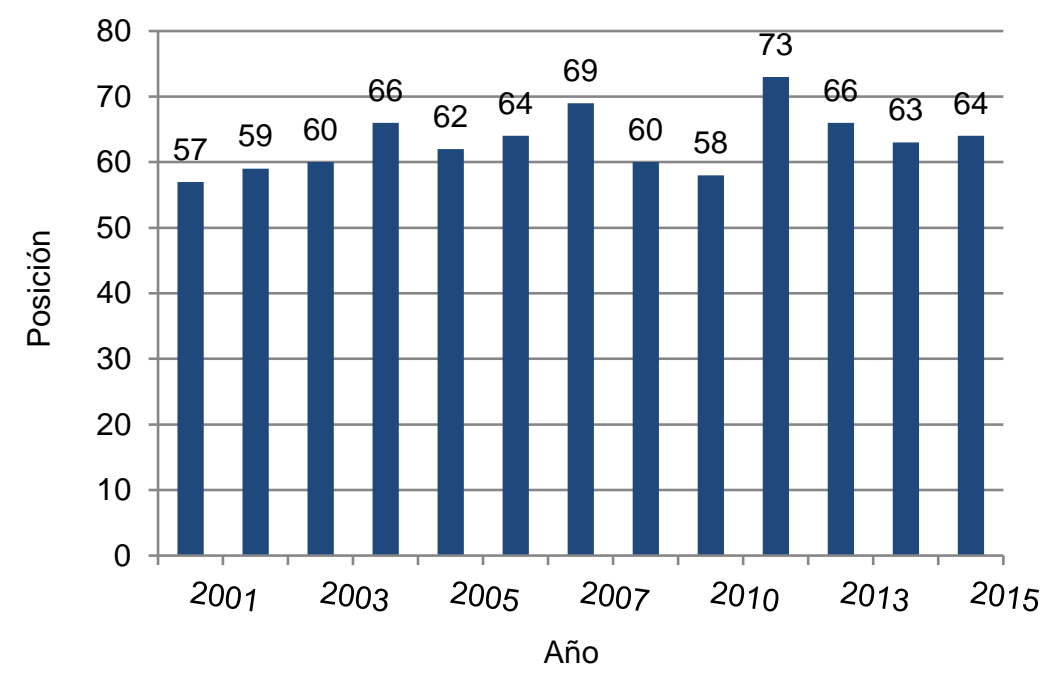

Fig. 1: Evolución de la posición IPC de Colombia.

En este punto cabe destacar la posición de la ITU (2015), con respecto a la accesibilidad a las comunicaciones digitales y a la web. Consideran que es un derecho humano y es crítico para todos los miembros de la sociedad, y en particular para las personas con discapacidad. Promueven que los gobiernos avanzan hacia e-gobiernos, donde sus esfuerzos se deben centrar en asegurar el acceso a Internet y la penetración de banda ancha en zonas rurales y urbanas así como en ofrecer diferentes servicios en línea. Igualmente afirman que en Latinoamérica, todos los países tienen alguna política sobre accesibilidad digital pero sin resultados satisfactorios. En el año 2014, esta misma organización ya había publicado un conjunto de herramientas para ayudar a los países en la formulación de sus políticas y reglamentaciones de las TIC relacionadas con su accesibilidad, de manera que se eliminaran las barreras al utilizar los servicios web. Los componentes de información y las interfaces de usuario recomendados por esta organización son: perceptibilidad, manejabilidad, comprensibilidad y robustez. Cada uno de estos componentes se describe en la Tabla 1, a continuación.

Tabla 1: Componentes de información y las interfaces de usuario recomendados por ITU (2015)

\begin{tabular}{|c|l|}
\hline Componente Web & \multicolumn{1}{c|}{ Significado } \\
\hline Perceptibilidad & $\begin{array}{l}\text { Los usuarios deben percibir la información que se les presenta, no puede ser invisible } \\
\text { a todos sus sentidos }\end{array}$ \\
\hline Manejabilidad & Los usuarios deben poder manejar todos los componentes de la información \\
\hline Comprensibilidad & Los usuarios deben comprender la información y el manejo de la interfaz \\
\hline Robustez & $\begin{array}{l}\text { Los usuarios deben poder acceder a los contenidos mismo con los avances de las } \\
\text { tecnologías }\end{array}$ \\
\hline
\end{tabular}

Países del primer mundo, utilizan portales electrónicos para que los ciudadanos tengan acceso a los servicios que presta el estado. En Estados Unidos, el portal GobiernoUSA.gov es el portal oficial del Gobierno en español, el cual ofrece servicios e información oficial de una manera práctica para el usuario. Proporciona a los hispanohablantes acceso a información, programas y servicios, artículos originales y los canales de redes sociales del Gobierno. Está específicamente diseñado para satisfacer las necesidades de información de los hispanohablantes de Estados Unidos y proporciona información oficial y centralizada del Gobierno de los Estados Unidos durante desastres naturales con el fin de que los usuarios puedan conocer acerca de los programas de asistencia para las víctimas. 


\section{AVANCES RECIENTES}

En Colombia, se dispone de unas normas de las más avanzadas en el área de lberoamérica, las cuales se encuentran en el decreto 1151 del 14 de abril de 2008 (Ministerio de Comunicaciones, 2008), donde se establecen los lineamientos generales de la Estrategia de Gobierno en Línea. Específicamente, en el artículo 3 , se establecen los principios, entre los que se encuentran: gobierno centrado en el ciudadano, visión unificada del estado, acceso equitativo y multicanal, protección de la información del individuo, credibilidad y confianza en el gobierno en línea. En el artículo 7 del mismo decreto, referido al Modelo de Gobierno en línea, se establecen sus componentes: a) información en línea, b) interacción en línea, c) transacción en línea, d) transformación, e) democracia en línea y f) elementos transversales. También se definen los niveles de madurez: inicial, básico, avanzado y mejoramiento permanente. En todo este proceso también se determinó la elaboración de los manuales y el software a utilizar. Es importante señalar que Colombia ha mostrado un crecimiento en el número de emprendimientos privados a través de organizaciones no gubernamentales y empresa privada, para contribuir con la disminución de la brecha digital de los ciudadanos y de esa manera incrementar la inclusión digital de todos los ciudadanos colombianos. No obstante estos esfuerzos, es importante el aporte desde la academia para fortalecer la iniciativa del Estado.

En Colombia existen emprendimientos para mejorar la accesibilidad educativa para personas con discapacidad. Uno de ellos es el presentado por la alcaldía de Medellín en el año 2012 a través del programa Medellín Digital, el cual realizaba el taller denominado Accesibilidad Digital de manera gratuita con el propósito de sensibilizar y enseñar a maestros, bibliotecarios y público en general. Estos talleres fueron diseñados para lograr la inclusión social de las personas en condición de discapacidad, quienes aprenden sobre la usabilidad y manejo de software especializado de accesibilidad para que se conviertan en canales de acceso al mejoramiento en la calidad de vida de discapacitados y adultos mayores. Igualmente, el Instituto Nacional para Ciegos ( $\mathrm{INCl}$ ) ha producido y distribuido información digital accesible para personas que presentan alguna discapacidad frente a los impresos (INCl, 2014). Colombia fue el primer país latinoamericano en comenzar a producir libros hablados digitales en formato DAISY (Digital Accesible Information System), posteriormente, Brasil se sumó a los países latinoamericanos en esta producción, pero Colombia sigue siendo el único de habla hispana en el continente.

En el año 2014, la Universidad de Antioquia con el apoyo del Ministerio de Tecnologías de la Información y las Comunicaciones (Universidad de Antioquia, 2014), desarrolló el proyecto Living Lab como una iniciativa para personas con discapacidad visual del Sistema de Bibliotecas de la Universidad de Antioquia, en el marco de la Convocatoria Crea Digital 2014, con el objetivo de apalancar una cultura de diseño accesible entre los generadores de contenidos digitales, contribuyendo con el enriquecimiento de los componentes del Ecosistema Digital del país. Esto permite a todas las personas, incluidas las personas con discapacidad, acceder a los recursos electrónicos: páginas web, software, dispositivos móviles, lectores de pantalla, facilitando con ello el uso de las comunicaciones digitales y por tanto el acceso a toda aquella información fundamental. En este proyecto se unen los esfuerzos de la academia, el Estado y la sociedad civil, para diseñar contenidos digitales accesibles, de manera que la información llegue a un mayor número de personas, especialmente a aquellas en situación de discapacidad. Con ello se incrementan sus posibilidades de participación e inclusión social y la construcción de una sociedad digital más responsable e incluyente.

En otro orden de ideas, y a manera de ejemplo del avance de las TIC en los diferentes ámbitos sociales, en el portal de Colombia Digital (2016) se encuentra un estudio desarrollado por Burson-Marsteller en el cual se muestra cómo la diplomacia digital encuentra su lugar en Twitter. En el mencionado estudio se muestra cómo los diferentes gobiernos alrededor del mundo y sus dirigentes usan Twitter de manera continua, aunque en Facebook tienen mayor cantidad de seguidores. La Tabla 2 refleja esta situación. De esta manera se puede observar cómo los gobiernos utilizan las redes sociales para conectarse e influir en audiencias clave a través de diferentes redes sociales. En el mismo estudio también se hace referencia a los líderes más exitosos en el uso de medios digitales: Presidente Barack Obama y el equipo de la Casa Blanca, Mauricio Macri, Presidente de Argentina y Justin Trudeau, Primer Ministro de Canadá.

De este análisis se puede inferir que a nivel de los diferentes países, cada vez más los representantes de los Estados utilizan las herramientas web para mantener contacto con los ciudadanos y la tendencia es utilizar cada vez más estas las plataformas, principalmente las más populares, entre los ciudadanos. Esta es una señal de que cada día es más importante para la ciudadanía capacitarse en el uso de las TIC's, para mantener una comunicación libre y directa con sus representantes a nivel del estado, comenzando con sus concejales, pasando por sus diputados, hasta llegar al presidente de la república y su equipo de ministros.

En cuanto al punto de vista educativo, el uso de las TIC es una realidad que se suma a la evolución en la educación, y ha motorizado la búsqueda de nuevas alternativas de comunicación entre los alumnos y profesores y ha transformando un modelo de educación estático en uno móvil y flexible. Este nuevo modelo 
ha sido apalancado por la innovación de la tecnología y ha sido adaptado a las necesidades del nuevo mundo con lo cual se amplía la oferta educativa en cuanto a la modalidad de interacción alumno-profesor y se asumen una serie de retos relacionados principalmente con: (a) La disponibilidad de equipos y conectividad de las diferentes clases sociales.(b) Las estrategias de enseñanza que deben cambiar debido a la falta de interacción entre alumno profesor, por la resistencia al cambio que se podría observar en cualquiera de los participantes. (c) Pocos recursos de video de alta calidad: algunos sistemas carecen de capacidades de grabación y difusión en vivo, lo que hace difícil compartir cursos y mejorar la calidad del video.

Tabla 2: Uso de redes sociales por parte de gobiernos mundiales.

\begin{tabular}{|c|c|}
\hline Red Social & Observación \\
\hline Twitter & $\begin{array}{l}\text { En los últimos cinco años Twitter ha sido un canal de comunicación preferido por los líderes } \\
\text { mundiales y los gobiernos. Lo usan jefes de Estado y los gobiernos de más de } 173 \text { países, } \\
\text { equivalentes al } 90 \% \text { de los Estados miembros de Naciones Unidas. Las cancillerías tienden a } \\
\text { usar Twitter para establecer relaciones mutuas. En mayo } 2015 \text {, el Departamento de Estado } \\
\text { de Estados Unidos usó Twitter para establecer contacto con su contraparte en Cuba, meses } \\
\text { antes del re-establecimiento de relaciones diplomáticas. }\end{array}$ \\
\hline Facebook & $\begin{array}{l}\text { Facebook es la segunda red más usada con } 169 \text { gobiernos con páginas oficiales. Sin } \\
\text { embargo, los seguidores en Facebook duplican a los seguidores en Twitter. }\end{array}$ \\
\hline Youtube & $\begin{array}{l}\text { Se ubica tercero en el ranking, siendo utilizado por } 78 \% \text { de los Estados miembros de las } \\
\text { Naciones Unidos. }\end{array}$ \\
\hline Instagram & Su uso alcanza al $70 \%$ de los gobiernos. \\
\hline Vine y Snapchat & $\begin{array}{l}\text { Preferido por Estados que cuentan con equipos digitales más grandes; apelan a audiencias } \\
\text { más jóvenes. }\end{array}$ \\
\hline $\begin{array}{l}\text { Periscope y } \\
\text { Facebook Live }\end{array}$ & $\begin{array}{l}\text { Gobiernos que no tienen grandes equipos comunicacionales, como sucede en América } \\
\text { Latina, aprovechan los beneficios de transmisión que ofrece para transmitir sus eventos de } \\
\text { prensa. }\end{array}$ \\
\hline
\end{tabular}

La evolución de esta modalidad de enseñanza aprendizaje depende, en gran parte, de mejorar los puntos mencionados anteriormente. El mismo escenario se presenta en el área empresarial. Las empresas ofrecen sus productos y servicios a través de portales web, donde sus productos van directamente de la empresa al usuario final, sin intermediarios. Esta dinámica contribuye a la disminución de tiempos y costos, reduciendo el costo del producto final. Para que sea posible esta comunicación, se debe contar con la conectividad y los equipos, y que los usuarios puedan acceder a estos servicios al ser capaces de utilizar los medios digitales. En este sentido, el gobierno tiene la responsabilidad de mejorar la conectividad en los diferentes poblados del país, para garantizar el acceso de los diferentes estratos sociales. Los servicios de internet deben ser accesibles a toda la población, para contribuir con la inclusión de todos los ciudadanos colombianos y con el acceso a la educación y a los servicios tanto de las empresas como del estado.

Continuando en el ámbito educativo, González (2004) expresa que la extensión universitaria tiene la función de desarrollar talento humano bien capacitado y comprometido para una mejor proyección e integración comunitaria, ya que el destinatario de su proceder es la sociedad entera, sin distingos ni discriminaciones. Para tal fin los programas de extensión deben concebirse, diseñarse y llevarse a cabo en diálogo constante con la comunidad y con un pleno conocimiento de la Universidad acerca de la problemática de la sociedad. Igualmente debe estar en la búsqueda de un marco legal promotor de la labor extensionista y evaluar continuamente el impacto de su acción social. Enfatiza además que de esa manera, la extensión universitaria se constituye en un instrumento promotor de los cambios en pro de una mejor sociedad, por lo que concluye que es imperativo incorporar actividades extensionistas dentro del currículo universitario, para que la extensión no tenga sólo el valor de una contribución aislada, individual o grupal, a la difusión del conocimiento.

Ortiz y Morales (2011) exponen que la interacción universidad-sociedad es la razón de ser de los programas de extensión en las diferentes universidades latinoamericanas. De este proceso debería ser responsable un equipo de trabajo que pertenezca a la estructura de las universidades, que permita una planeación y una ejecución coordinada con las otras dos funciones: la docencia y la investigación, para de esa manera relacionarse con el quehacer de la Universidad y su forma de contribuir con solucionar los problemas de la sociedad. Sin embargo, existe una gran cantidad de experiencias en las universidades latinoamericanas, que muestran los diferentes caminos escogidos y de los que se pueden aprender valiosas lecciones. Estas diferentes rutas seleccionadas están estrechamente relacionadas con el entorno social de la Universidad. Un ejemplo de esto es que en las universidades latinoamericanas, y específicamente en las colombianas, desarrollan actividades de extensión universitaria en los tres enfoques: empresarial, social o cultural, y que su grado de desarrollo depende de factores del entorno regional y de los propósitos particulares de las 
instituciones universitarias, pero muy particularmente el desarrollo de la extensión desde el enfoque empresarial. Los autores anteriormente citados, concluyen que las políticas de extensión no pueden ser estáticas y deben adaptarse a su entorno, las cuales deben ser asumidas por toda la comunidad universitaria. Además el conocimiento desarrollado en las universidades debe ser apropiado por su entorno sin descuidar la razón de ser de la extensión.

Ahora bien, según lo expresado por Baggio (2013), fundador del Comité para la Democratización de la Información (CDI), en Colombia las condiciones están dadas para que sea un modelo de inclusión digital debido al rápido aumento en los niveles de acceso a internet, al incremento en la banda ancha, así como a las políticas públicas de inclusión digital, la construcción de infraestructura y un Ministerio de Tecnologías de la Información y Comunicación (MinTic, 2014) muy activo que empodera a los ciudadanos para catalizar el desarrollo del país. También considera que Colombia puede llegar a ser pionera en la puesta en funcionamiento de políticas de inclusión tecnológica disruptiva. El mismo autor señala, además, que los esfuerzos se deberían enfocar en las comunidades de bajos ingresos, preparándolos para utilizar la tecnología como una herramienta para crear sus propios emprendimientos, una cultura ciudadana y aumentar la competitividad de las personas para que puedan ingresar al mercado laboral. También manifiesta el rol de las empresas en este propósito, pues éstas deben estimular a sus empleados para el uso responsable de la tecnología y no como un espejo de la sociedad de consumo.

La Convención de la ONU sobre los Derechos de las Personas con Discapacidad brindó en el año 2016, un reconocimiento internacional a Tecno ayudas, empresa colombiana con sede en Antioquia, por su participación como empresa del sector privado en el desarrollo de la inclusión digital ciudadana en Colombia. Tal reconocimiento internacional se realizó gracias al kit de inclusión digital que diseñaron, conformado por un conjunto de herramientas y capacitación para la inclusión digital. Este kit está compuesto por dispositivos para acceso al computador, tales como: mouse, teclado y pulsador, y en el caso del kit de accesibilidad para bibliotecas, se implementan otros elementos para el acceso a la lectura. Otra empresa colombiana que contribuye al proceso de inclusión digital es Telefónica (Fundación Telefónica, 2008), que conjuntamente con el gobierno conjugan esfuerzos para cerrar la brecha informática existente debido a que la penetración de computadores en Colombia es la más baja de América Latina (6\%).

Por su parte, la Fundación Marina Orth con el apoyo de Microsoft Colombia, ha implementado un modelo de pedagogía con nuevas oportunidades en la tecnología llamado proyecto StepbyStep. El proyecto desarrolla programas en tecnología y lenguaje para que los niños de los poblados de la región de Antioquia, tengan la oportunidad de continuar con estudios universitarios o capacitaciones tecnológicas y para que los egresados puedan tener un empleo remunerado dentro de la economía del país. En el año 2015, la misma fundación presentó el proyecto SparkTic para atender a la población NiNi (jóvenes que ni estudian ni trabajan) que han desertado del sistema escolar formal para así contribuir con el desarrollo de habilidades tecnológicas en este sector de la población colombiana.

Como se puede observar, la inclusión digital no es una tarea fácil. Requiere de mucho esfuerzo de parte de todos los grupos de interés y ha de ser fomentada principalmente por el Estado, ya que involucra educación y conocimiento de las TIC y éstas, a su vez, requieren de plataformas tecnológicos para su uso. Este tipo de proyectos demanda grandes inversiones monetarias que pueden ser apoyadas por la empresa privada y los entes educativos. Estos últimos serían el enlace entre el ciudadano y las TIC, sumando esfuerzos para la reducción de la brecha digital. En este sentido, desde el gobierno se están realizando esfuerzos para llegar a los más pobres y a los más alejados. Ejemplo de ello es el despliegue del Proyecto Nacional de Fibra Óptica, una gran autopista que permitirá llevar Internet de alta velocidad al 96\% del país, convencidos de que un país cada vez más y mejor interconectado tiene un inmenso potencial para generar riqueza y desarrollo socioeconómico. El desafío de masificar el uso de Internet en todos los rincones del país tiene la finalidad de generar empleo, disminuir la pobreza y aumentar la competitividad, para lograr la prosperidad y mejores condiciones de vida para los colombianos.

Es en este ámbito y considerando los avances de la tecnología, que la extensión universitaria podría encontrar una oportunidad de capacitar a los ciudadanos en el uso de las TIC's, para incorporarlos a la era digital. Igualmente es una oportunidad para socializar el resultado de sus investigaciones entre los miembros de la comunidad.

\section{METODOLOGÍA}

La metodología utilizada en la presente investigación se tipifica como descriptiva con un diseño de investigación transeccional-descriptivo y de campo según los planteamientos de Hernández et al., (2010) y Arias (2012). Para iniciar la investigación, se realizó una revisión de bibliografía relacionada con las dimensiones del estudio: accesibilidad digital e inclusión digital, además de consultar temas relacionados con las TIC, los cuales contribuyeron en el desarrollo del tema en análisis. Estas fuentes consultadas fueron utilizadas como referente teórico y como base para el análisis de los resultados. 


\section{Procedimiento para la recolección de información}

A partir de la revisión documental, se diseñó un instrumento de recolección de información tipo cuestionario el cual fue sometido apruebas de validez por parte de expertos. La confiabilidad fue medida por medio del coeficiente de Alfa de Cronbach, arrojando un resultado de 0.85 , lo cual indica que el instrumento es altamente confiable, según lo estipulado por Ruiz (1998). El mismo estuvo conformado por 9 preguntas de las cuales 4correspondían a la accesibilidad y las 5 restantes a inclusión digital. Dicho cuestionario contó con una escala tipo Lickert de 5 opciones de respuestas a las cuales se le asignaron los siguientes valores: muy alta $=5$, alta= 4 , oderado= 3 , baja $=2$ y muy baja $=1$.

Esta escala sirvió para medir el grado de acuerdo o desacuerdo de los ciudadanos colombianos con respecto al planteamiento de que desde las universidades se mejoren los índices de accesibilidad e inclusión digital de los ciudadanos con el resto de los sectores clave de la sociedad colombiana. Los intervalos utilizados para calcular la media de los resultados se presentan en la Tabla 3.

Tabla 3: Intervalos para la interpretación de la media

\begin{tabular}{|l|l|l|l|}
\hline \multicolumn{1}{|c|}{ Valor } & \multicolumn{1}{|c|}{ Intervalos } & \multicolumn{1}{c|}{ Alternativas } & \multicolumn{1}{c|}{ Categoría } \\
\hline 5 & $4,21-5$ & Totalmente de acuerdo & Muy Alto \\
\hline 4 & $3,41-4,2$ & De acuerdo & Alto \\
\hline 3 & $2,61-3,4$ & Medianamente de acuerdo & Moderado \\
\hline 2 & $1,81-2,6$ & En desacuerdo & Bajo \\
\hline 1 & $1-1,8$ & Totalmente en desacuerdo & Muy Bajo \\
\hline
\end{tabular}

Población y muestra

La población de esta investigación estuvo constituida por 846.609 habitantes del Departamento de la Guajira. La muestra fue de tipo probabilística y estuvo conformada por 400 sujetos entre 18 y 50 años de edad, sin importar su nivel educativo y social. Esta muestra se obtuvo, aplicando la ecuación (1) propuesta por Arias (2012):

$$
\mathrm{N}=\frac{N \cdot Z_{c}^{2} \cdot p \cdot q}{(N-1) \cdot e^{2}+Z_{c} \cdot p \cdot q}
$$

Donde $\mathrm{N}=$ total de elementos que integran la población: 846.609 individuos; $\mathrm{Zc}=$ Zeta crítico o valor determinado por el nivel de confianza adoptado. Para un nivel de confianza de $95 \%$ es igual a 2, para un nivel de confianza del $99 \%$ es igual a 3 ; $E$ = error muestral que oscila entre $1 \%$ y $5 \%$. En este caso se utilizó $5 \%$; $p$ $=$ proporción de elementos que presentan una determinada característica a ser investigada. En este caso 50; y q=proporción de elementos que no presentan la característica a ser investigada, se aplica p+q=1.

\section{Procedimiento para el análisis de los datos}

Una vez que se recolectaron todas las encuestas, se procedió a construir una hoja de cálculo en Excel 2010, donde se cargaron las respuestas de los encuestados. Los resultados se expresaron en términos de frecuencias absolutas y relativas, utilizando la media como medida de tendencia central. Posteriormente, se graficaron utilizando histogramas de frecuencias para su análisis.

\section{RESULTADOS Y DISCUSIÓN}

A continuación se mostrara el resultado del cuestionario el cual estuvo conformado por 4 preguntas que correspondían a la accesibilidad y otras 5 a la inclusión digital. Dicho cuestionario contó con una escala tipo Lickertde 5 opciones de respuestas las cuales fueron mencionadas en el procedimiento para la recolección de información

\section{Accesibilidad}

En la Tabla 4, se presentan preguntas correspondientes a la dimensión Accesibilidad. En la Tabla 5 se encuentran los resultados para las frecuencias absolutas, relativas y la media aritmética para cada una de las preguntas y de la dimensión. Con estos datos se elabora un histograma de frecuencias y se obtiene la Figura 2 , en la cual se observa una amplia tendencia hacia la categoría de Muy Alto, mientras que las categorías restantes se observan con muy bajo número de respuestas. 
Tabla 4: Distribución de las preguntas para la dimensión accesibilidad.

\begin{tabular}{|c|c|}
\hline № & Preguntas \\
\hline 1 & $\begin{array}{l}\text { ¿La educación universitaria en Colombia debería dotar de habilidades digitales a los ciudadanos para } \\
\text { mejorar sus niveles de accesibilidad? }\end{array}$ \\
\hline 2 & ¿La formación en TIC desde la Universidad garantiza la accesibilidad digital de los ciudadanos? \\
\hline 3 & $\begin{array}{l}\text { ¿La formación en TIC desde la Universidad, debe incluir programas especiales para personas } \\
\text { discapacitadas, para mejorar sus niveles de accesibilidad digital? }\end{array}$ \\
\hline 4 & $\begin{array}{l}\text { ¿Los programas tecnológicos implementados por las universidades colombianas, contribuyen con la } \\
\text { mejora de las condiciones de vida de los ciudadanos? }\end{array}$ \\
\hline
\end{tabular}

Tabla 5: Distribución de frecuencias absolutas (Fa) y relativas (Fr) sobre la dimensión accesibilidad

\begin{tabular}{|c|c|c|c|c|c|c|c|c|}
\hline Pregunta & \multicolumn{2}{|c|}{1} & \multicolumn{2}{|c|}{2} & \multicolumn{2}{|c|}{3} & \multicolumn{2}{c|}{4} \\
\hline Respuesta & Fa & Fr (\%) & Fa & Fr (\%) & Fa & Fr (\%) & Fa & Fr (\%) \\
\hline 1 & 22 & 5,50 & 17 & 4,25 & 16 & 4,00 & 30 & 7,50 \\
\hline 2 & 25 & 6,25 & 25 & 6,25 & 22 & 5,50 & 41 & 10,25 \\
\hline 3 & 42 & 10,50 & 41 & 10,25 & 35 & 8,75 & 56 & 14,00 \\
\hline 4 & 84 & 21,00 & 89 & 22,25 & 93 & 23,25 & 75 & 18,75 \\
\hline 5 & 227 & 56,75 & 228 & 57,00 & 234 & 58,50 & 198 & 49,50 \\
\hline sumatoria & 400 & 100 & 400 & 100 & 400 & 100 & 400 & 100 \\
\hline$(\bar{X})$ & 4,17 & \multicolumn{9}{|c|}{4,22} & \multicolumn{3}{|c|}{4,27} & \multicolumn{2}{c|}{3,93} \\
\hline Dimensión & \multicolumn{9}{|c|}{45} \\
\hline
\end{tabular}

4. ¿Los programas tecnológicos implementados por las universidades colombianas, contribuyen en la mejora de las condiciones de vida de los ciudadanos? 3. ¿La formación en tic's desde la universidad, debe incluir programas especiales para personas discapacitadas, para mejorar sus niveles de accesibilidad digital?

2. ¿La formación en tic's desde la universidad, garantiza la accesibilidad digital de los ciudadanos?

1. ¿La educación universitaria en Colombia debería dotar de habilidades digitales a los ciudadanos para mejorar sus niveles de accesibilidad?

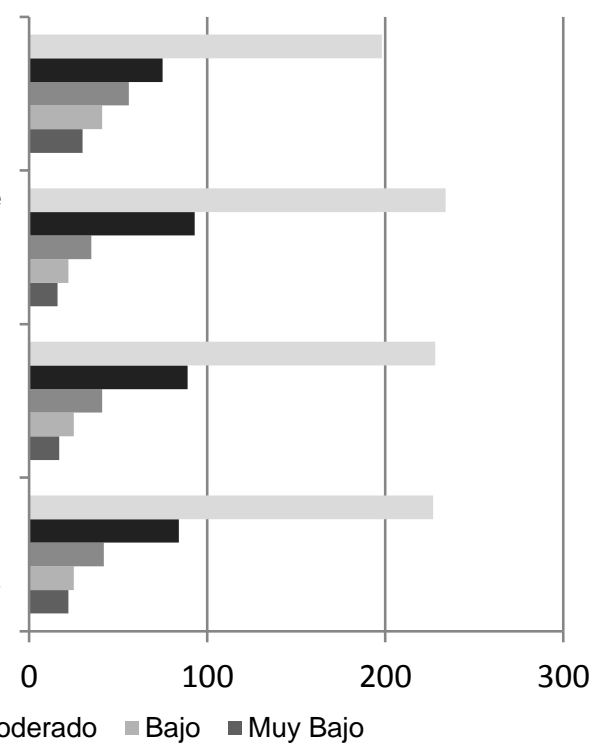

Fig. 2: Distribución de frecuencias absolutas de los resultados sobre accesibilidad

Puede observarse en la Tabla 5 que la media de la dimensión accesibilidad resultó en 4,15 ubicándose en la categoría de Alto. Esto significa que la mayoría de los encuestados está de acuerdo con que la academia debe convertirse en el principal ente formador para garantizar la accesibilidad de los ciudadanos a los sectores clave de Colombia. Estos resultados concuerdan con lo expresado por García et al., (2008), para quienes un entorno es plenamente accesible cuando en el mismo se puede avanzar en todos sus caminos, de forma autónoma y para ello recomiendan crear una serie de estrategias políticas o sociales de modo que la accesibilidad sea universal o integral. Igualmente, la ITU (2015) considera que la accesibilidad a la web es crítica para todos los miembros de la sociedad por lo que es necesario que los gobiernos garanticen el acceso a Internet, la penetración de banda ancha en zonas rurales y urbanas y la oferta de diferentes servicios en línea.

\section{Inclusión digital}

Al igual que en la dimensión anterior, las preguntas para la dimensión inclusión digital se muestran en la Tabla 6. En la Tabla 7, se encuentran los resultados para las frecuencias absolutas, relativas y media aritmética. Para continuar con el análisis, con los datos de la Tabla 6 , se elaboró un histograma de frecuencias y se obtiene la Figura 3. En esta figura, al igual que en la dimensión anterior, se observa una amplia tendencia hacia la categoría 
Muy Alto, mientras que las categorías restantes se observan con muy bajo número de respuestas. En esta Tabla (Tabla 7) puede observarse que la media $(X)$ resultante se ubicó en 4,25, ubicándose la dimensión Inclusión digital en la categoría de Muy Alto. Esto significa que la mayor parte de los encuestados opinan que desde la Universidad se pueden mejorar los niveles de inclusión digital de los ciudadanos colombianos, a través del desarrollo de sus competencias tecnológicas y que, además, los programas a desarrollar deben estar basados en las exigencias actuales en cuanto al uso de las TIC. Coinciden igualmente en que la formación ofrecida desde las universidades debe incluir a la comunidad en general, independientemente del nivel académico.

Tabla 6: Distribución de frecuencias absolutas para la dimensión inclusión digital.

\begin{tabular}{|c|l|}
\hline$N^{0}$ & \multicolumn{1}{|c|}{ Preguntas } \\
\hline 1 & $\begin{array}{l}\text { ¿La universidad es el ente desde donde se deben desarrollar programas para la inclusión } \\
\text { digital ciudadana? }\end{array}$ \\
\hline 2 & $\begin{array}{l}\text { ¿Desde la universidad debería manejar la formación digital de todos los ciudadanos, } \\
\text { independientemente de su nivel académico? }\end{array}$ \\
\hline 3 & $\begin{array}{l}\text { ¿Los programas de preparación universitaria colombiana deben estar vinculados con las } \\
\text { exigencias actuales en cuanto al uso de las TIC? }\end{array}$ \\
\hline 4 & $\begin{array}{l}\text { ¿La educación universitaria colombiana debe contribuir con el desarrollo de las } \\
\text { competencias tecnológicas del ciudadano? }\end{array}$ \\
\hline
\end{tabular}

Tabla 7: Distribución de frecuencias absolutas (Fa) y relativas (Fr) sobre la dimensión inclusión digital

\begin{tabular}{|c|c|c|c|c|c|c|c|c|c|c|}
\hline Pregunta & \multicolumn{2}{|c|}{1} & \multicolumn{2}{|c|}{2} & \multicolumn{2}{|c|}{3} & \multicolumn{2}{|c|}{4} & \multicolumn{2}{|r|}{5} \\
\hline Respuesta & $\mathrm{Fa}$ & $\operatorname{Fr}(\%)$ & $\mathrm{Fa}$ & $\operatorname{Fr}(\%)$ & $\mathrm{Fa}$ & $\operatorname{Fr}(\%)$ & $\mathrm{Fa}$ & $\operatorname{Fr}(\%)$ & $\mathrm{Fa}$ & $\operatorname{Fr}(\%)$ \\
\hline 1 & 17 & 4,25 & 19 & 4,75 & 16 & 4,00 & 21 & 5,25 & 18 & 4,50 \\
\hline 2 & 25 & 6,25 & 23 & 5,75 & 24 & 6,00 & 28 & 7,00 & 27 & 6,75 \\
\hline 3 & 31 & 7,75 & 29 & 7,25 & 27 & 6,75 & 33 & 8,25 & 38 & 9,50 \\
\hline 4 & 99 & 24,75 & 82 & 20,50 & 84 & 21,00 & 92 & 23,00 & 83 & 20,75 \\
\hline 5 & 228 & 57,00 & 247 & 61,75 & 249 & 62,25 & 226 & 56,50 & 234 & 58,50 \\
\hline sumatoria & 400 & 100 & 400 & 100 & 400 & 100 & 400 & 100 & 400 & 100 \\
\hline$(\overline{\mathrm{X}})$ & \multicolumn{2}{|c|}{4,24} & \multicolumn{2}{|c|}{4,29} & \multicolumn{2}{|c|}{4,32} & \multicolumn{2}{|c|}{4,19} & \multicolumn{2}{|c|}{4,22} \\
\hline$(\bar{X})$ Dimensión & \multicolumn{10}{|c|}{4,25} \\
\hline
\end{tabular}

Esta necesidad concuerda con lo expresado por López y Samek (2009), quienes consideran que se deben desarrollar proyectos de inclusión digital que incluyan a todas las personas de la sociedad, principalmente a las que enfrentar mayores niveles de exclusión social. En este sentido, Baggio (2013) expone que Colombia tiene el potencial necesario para ser pionero en la implementación de una política de inclusión tecnológica disruptiva, debido al rápido aumento en los niveles de acceso a internet, un aumento en la banda ancha y las políticas públicas de inclusión digital, enfocándose en las comunidades de bajos ingresos, con el propósito de prepararlos para crear su propia salida de la banda de pobreza. Se pudo observar en el análisis de las dos dimensiones en estudio, que la mayoría de los ciudadanos colombianos ven la necesidad de que la universidad lidere la formación de la sociedad en el uso de las TIC para mejorar los niveles de accesibilidad e inclusión digital, contribuyendo así con el desarrollo del país.

9. ¿Considera que la universidad debe ofrecer a la comunidad en general, formación en las tic's, para.

8. ¿La educación universitaria colombiana debe contribuir con el desarrollo de las competencias.

7. ¿Los programas de preparación universitaria colombiana deben estar vinculados con las...

6. ¿desde la universidad debería manejar la formación digital de todos los ciudadanos, independientemente...

5. ¿la universidad es el ente desde donde se deben desarrollar programas para la integración digital...

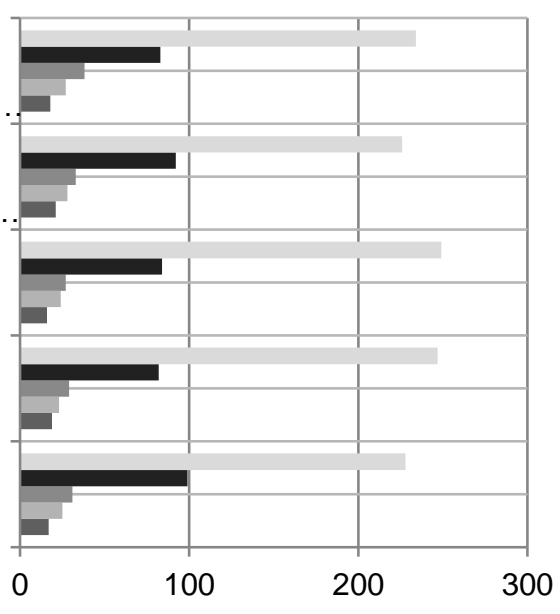

Muy Alto $\square$ Alto $\square$ Moderado $\square$ Bajo $\square$ Muy Bajo

Fig. 3: Distribución de frecuencias absolutas de los resultados sobre inclusión digital 


\section{Programas de extensión universitaria}

En función de los resultados presentados, donde la mayor parte de los encuestados opina que desde la universidad se deben fortalecer las capacidades digitales de la población y que ésta debe ser líder en la formación de la sociedad, independientemente de la condición social, nivel educativo y discapacidad de las personas a ser formadas, se presenta una serie de recomendaciones para hacer posible esta situación.

Estas acciones deben ser capitalizadas desde la extensión universitaria, la cual en primer lugar, se debe responsabilizar de este proceso a un equipo de trabajo que pertenezca a la estructura de las universidades, que permita una planeación y una ejecución coordinada con las otras dos funciones: la docencia y la investigación, para de esa manera relacionarse con el quehacer de la Universidad. Esta estructura estará en capacidad de realizar las investigaciones necesarias con el apoyo de los decanatos de pre y post grado, para realizar la segunda acción propuesta.

Como segunda acción se plantea promover la realización de investigaciones para diagnosticar: (i) Los niveles de formación de la ciudadanía en el uso de las TIC; (ii) Cuáles son las capacidades tecnológicas que necesitan desarrollar los ciudadanos, en función de su relación con los sectores clave de la sociedad; (iii) Cuál es la capacidad de las universidades para formar al resto de la sociedad en cuanto a recurso humano, técnico y financiero; y (iv) Cuáles serían los programas a impartir según los estratos sociales y determinar cuáles requieren más formación.

La tercera acción consiste en utilizar los resultados del diagnóstico como insumo para definir los programas a ofrecer, diseñar los programas a dictar, captar y desarrollar el capital humano para así proceder a la búsqueda de los fondos para financiar estas iniciativas.

Por último, se planificarían los cursos o talleres para que estén accesibles a la comunidad. Para mantenerse en el tiempo, es necesario que tanto los profesores o facilitadores de los cursos como los programas a impartir estén actualizados. Este punto representa un reto para estructuras tan estrictas como las universitarias, donde el desarrollo y preparación de programas, no avanza a la misma velocidad de las innovaciones tecnológicas. Es importante destacar que los cursos y talleres deben contemplar la inclusión de personas discapacitadas, igualmente utilizar diferentes estrategias para llegar a todas partes como el uso de aulas virtuales, donde los ciudadanos puedan participar, independientemente de la distancia entre la universidad.

\section{CONCLUSIONES}

Del análisis documental realizado se concluye que la extensión universitaria es el componente de la academia que cumple la función de relacionarse con el entorno y que tiene la capacidad de llevar conocimientos a todos los integrantes de la sociedad.

Una vez culminado el análisis de los datos, se concluye que la mayor parte de los ciudadanos opina que la Universidad es el ente desde el cual se debe liderar la capacitación de los ciudadanos para mejorar sus habilidades digitales, para mejorar los niveles de accesibilidad e inclusión digital, para relacionarlo con los factores clave de la sociedad, contribuyendo así a construir una sociedad más inclusiva, con equidad y que contribuya a fomentar los valores democráticos.

Igualmente los encuestados opinan esta capacitación debe ser accesible a todos los ciudadanos, independientemente de su nivel académico y social.

\section{REFERENCIAS}

Agustín M. y M. Clavero, Indicadores sociales de inclusión digital: brecha y participación ciudadana, (en línea: https://goo.gl/Fe75Zt, acceso: 26 de junio 2016), España (2009)

Arias, F., El proyecto de Investigación, 6a Edición, 30-38, Editorial Episteme, Caracas, Venezuela (2012)

Baggio R., Colombia podría ser un modelo de inclusión digital, (en línea: https://goo.gl/xOaurm, acceso: 15 de abril 2016), Colombia (2013)

Cabezudo, N., Inclusión digital: perspectivas y experiencias, Zaragoza, Universidad de Zaragoza (2011)

Colombia Digital, Twiplomacy, Colombia (2016)

DNP (Departamento Nacional de Planeación) Empresarial, Reporte Global de Tecnologías de la Información (2015) 
Duarte, E., Inclusión digital, tres conceptos clave: conectividad, accesibilidad, comunicabilidad, Ar@cne, Revista electrónica de recursos en Internet sobre Geografía y Ciencias Sociales, 150 (2011)

Fundación Telefónica, Inclusión Digital y Telecentros en América Latina, Editorial Ariel, Madrid, España (2008)

García, A., I. González y B. Ruiz, El concepto de accesibilidad universal (en la web: https://goo.gl/68uKjp, acceso: 28 de diciembre 2016), Universidad Carlos II, Madrid, España (2008)

González, E., La Misión Académica Extensión Universitaria como Promotora de la Interacción Universidad Sociedad, Universidad Central de Venezuela, Docencia Universitaria, 5(1 y 2), 9-33 (2004)

Hernández R., P. Fernández y L. Baptista, Metodología de la Investigación, 5a edición, McGrawHill/Interamericana Editores, S.A. de C.V., México D.F., México (2010)

INCI (Instituto Nacional para Ciegos), Colombia es líder latinoamericano en producción de libros accesibles (en la web: https://goo.gl/30ycCw, acceso: 28 de marzo 2016), Colombia (2014)

ITU (International Telecommunication Unit), Américas Accesible II: Información y Comunicación para todos (en línea: https://goo.gl/D5XEDb, acceso: 18 de julio 2016), Colombia (2015)

López P. y T. Samek, Inclusión Digital: Un Nuevo Derecho Humano, Educación y Biblioteca, 172(1), 114-118 (2009)

Ministerio de Comunicaciones, Decreto 1151 de 2008: Por el cual se establecen los lineamientos generales de la Estrategia de Gobierno en Línea de la Republica de Colombia, se reglamenta parcialmente la Ley 962 de 2005, y se dictan otras disposiciones, 1-3, Bogotá, Colombia (2008)

MinTIC (Ministerio de Tecnologías de la Información y las Comunicaciones), Empoderar al ciudadano para interactuar con el Estado mediante las TI, (en la web: https://goo.gl/yd6oA7, acceso: 8 de julio 2016), Colombia (2014)

Mochi, P., Programas para la inclusión digital y la concertación de actores en procesos de desarrollo territorial, Polis, 8(1), 177-212 (2012)

Ortiz, M. y M. Morales, La extensión universitaria en América Latina: concepciones y tendencias, Educación y Educadores, ISSN 0123-1294, (en línea: https://goo.gl/U11ier, acceso: 18 de julio 2016), 14(2), 349-366 (2011)

Ramírez J., D. Rincón y M. Romero, Gobierno electrónico: un signo de inclusión digital y poder popular, Revista de Ciencias Sociales, ISSN 1315-9518, (en línea: https://goo.gl/5QlyrY, acceso: 18 de agosto 2016) 16(4), 709-720 (2010)

Ruiz, C., Instrumentos de Investigación Educativa, Ediciones CIDEG, C.A., Barquisimeto, Venezuela (1998)

Sequera, J., Una visión sobre extensión universitaria, Universidad Nacional Experimental de los Llanos Occidentales "Ezequiel Zamora" (en la web: https://goo.gl/6W1b8x, acceso: de diciembre 2016), Venezuela (2011)

Torres, J., La fundamentación del derecho a la inclusión digital, Revista Prolegómenos Derechos y Valores: 18(36), 47-64 (2015)

Universidad de Antioquia, Living Lab (en línea: https://goo.gl/HIQ8tn, acceso: 23 de agosto 2016), Colombia (2014) 